\title{
A rare case of yolk sac tumor of the vagina: Cytology and histopathological findings
}

\author{
Anil Dev Pant, Geeta Sayami, Viswanath Prasad, Anjan Shrestha \\ Department of Pathology, \\ Tribhuvan University Teaching Hospital, Kathmandu, Nepal
}

\begin{abstract}
:
Yolk sac tumors, the most common germ cell tumor in young infants and children, however, are distinctly uncommon site in the vagina.

A baby with bleeding per vaginum since one month presented at 9 months of age with raised Serum $\alpha$ fetoprotein (AFP) but normal levels of B-HCG and CA-125 is reported. Fine needle aspiration from the mass yielded material suggestive of a germ cell tumor. The histopathological findings further confirmed the diagnosis of a yolk sac tumor.
\end{abstract}

Key words: yolk sac tumor, endodermal sinus tumor, pediatric, vagina, germ cell tumor, $\alpha$-fetoprotein (AFP)

\section{Introduction}

Malignant germ cell tumors account for about 3\% of pediatric malignancies and among these tumors, yolk sac tumors account for most of the cases. Yolk sac tumors are rare, highly malignant germ cell tumors that usually involve children less than 3 years of age. ${ }^{1,2}$ Aberrant migration of germ cells during early embryonic life has been implicated as a possibility of origin. Clinical presentation is with a polypoid vaginal mass, bloody discharge, or sometimes even urinary retention if the bladder is compressed. ${ }^{3}$

These tumors may occur in pure form or a part of mixed germ cell tumors. The tumor was previously called mesonephroma ovarii because of the presence of glomeruli-like structures called Schiller Duval bodies. Thus the prognosis has changed significantly due to the effective chemotherapy, which reduces the tumor burden and the extent of the surgical resection.

\section{Case:}

A 9 month old baby girl presented to Gynecology Department, Tribhuvan University Teaching Hospital with bleeding per vaginum since 1 month along with passage of flakes of tissues. No palpable mass was found on the abdominal examination however, MRI showed a mass in the uterus and adnexae. Serum AFP was raised but $\mathrm{a}-\mathrm{HCG}$ and $\mathrm{CA}-125$ were within normal limits.

\section{Cytology}

Ultrasound guided fine needle aspiration cytology (FNAC) was performed from the vaginal mass and sent for examination to the Department of Pathology, T.U. Teaching Hospital. The smears were highly cellular, tumor cells showing mild to moderate atypia which were arranged in clusters and fragments, some singly scattered at places as well. Nuclei were round to oval and few showed prominent nucleoli. The cytoplasm of these cells was basophilic to vacuolated. Hyaline material, inflammatory cells and necrosis were also noted in the background. Since adenocarcinomas are quite unlikely at this age, the diagnosis of a germ cell tumor was made initially with the possibility a yolk sac tumor.(Fig. 1,2)

\section{Histopathology}

The biopsy specimen obtained from debulking surgery of pelvic and vaginal mass which grossly consisted of multiple pieces of grayish-brown tissue measuring $7 \mathrm{x}$ $3 \times 1.5 \mathrm{~cm}$ in total were sent for examination.

Histopathologic examination showed tumor composed of cells arranged mainly in reticular pattern characterized by a loose meshwork of communicating spaces. These tumor cells showed eosinophilic to clear cytoplasm with round to oval, hyperchromatic, paracentral to eccentric nuclei. Few nuclei also showed prominent nucleoli. Hyaline fragments and areas showing microcystic and macrocystic patterns were noted as well. Neural 


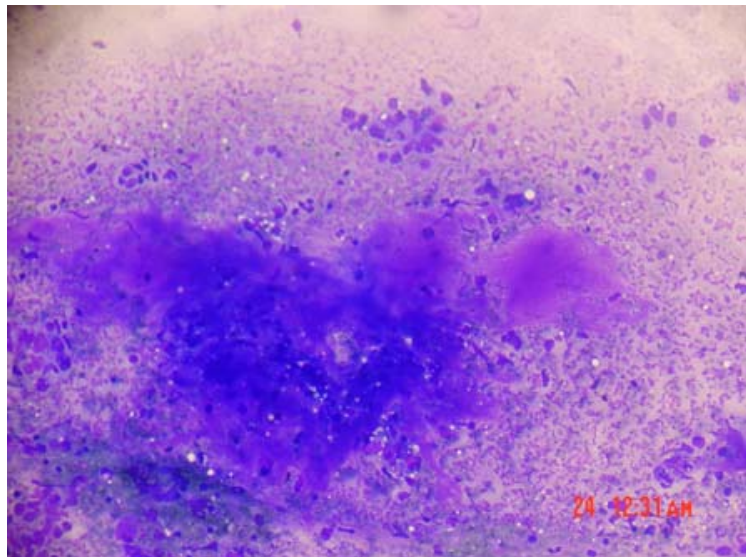

Fig 1:FNAC smears showing presence of tumor cells and hyaline stromal fragments admixed with each other (low power view - 10x)

invasion and invasion into part of endocervix and myometrium were also seen (Fig 3-5). No necrosis was seen.

\section{Discussion}

Cytologic findings include highly cellular smears with tumor cells arranged in clusters and acinar structures. These cells typically show moderate pleomorphism, and hyperchromatic nuclei with moderate amount of cytoplasm occasionally showing cytoplasmic vacuolations. Tumor cells have high $\mathrm{N}$ : $\mathrm{C}$ ratio, ovoid to round, moderately irregular, hyperchromatic nuclei and cytoplasmic vacuoles displacing the nuclei eccentrically, at places giving the impression of a mucin secreting adenocarcinoma. ${ }^{4}$

Various histopathologic subtypes of yolk sac tumor have been described in WHO fascicles of Pathology. They include reticular (microcystic), macrocystic, endodermal sinus type, papillary, solid, glandular,

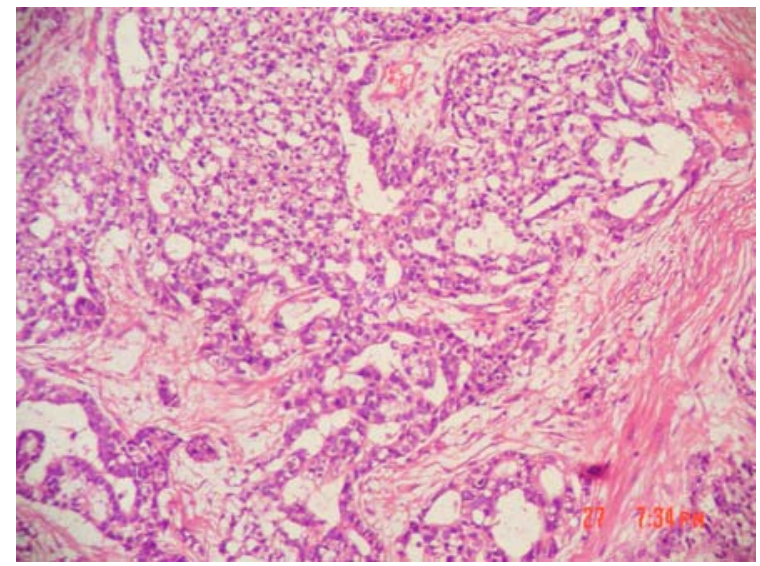

Fig 3 of yolk sac tumor showing various patterns, ncluding diffuse, glandular, and trabecular patterns. (low power $10 x)$

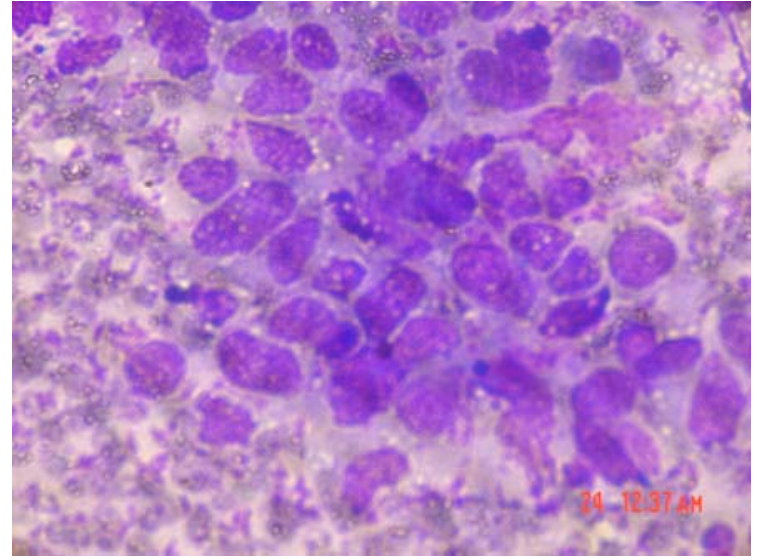

Fig 2: High power (40x) FNAC photomicrograph showing moderately atypical cells with hyperchromatic nuclei and scant to moderate cytoplasm arranged in a cluster.

myxomatous, sarcomatoid, polyvesicular, hepatoid and parietal types. Periodic acid Schiff's stain (PAS) can be used to stain the hyaline material and shows positivity which is resistant to digestion by diastase..$^{5}$

An AFP level estimation is mandatory in all young girls presenting with bleeding per vaginum or mass protruding through the vagina. ${ }^{3}$

Serum AFP can be used in the preoperative diagnosis, to monitor therapy effectiveness, and to detect recurrences before the clinical manifestations appear. Although experience with the use of serum AFP as a tumor marker in children is limited, recent data from large series of adults indicate disturbingly high incidence of false negative results (20-40\%) as reported by Nishat et al. Initially, in few cases, AFP levels were low suggest a possibility of other diagnoses. ${ }^{3}$ Levels of serum AFP ranged from lowest value of $382 \mathrm{IU} / \mathrm{mL}$ to highest $18,052 \mathrm{IU} / \mathrm{mL}$ (normal level $<30 \mathrm{IU} / \mathrm{mL}$ ) in a study by Kumar et al. ${ }^{1}$

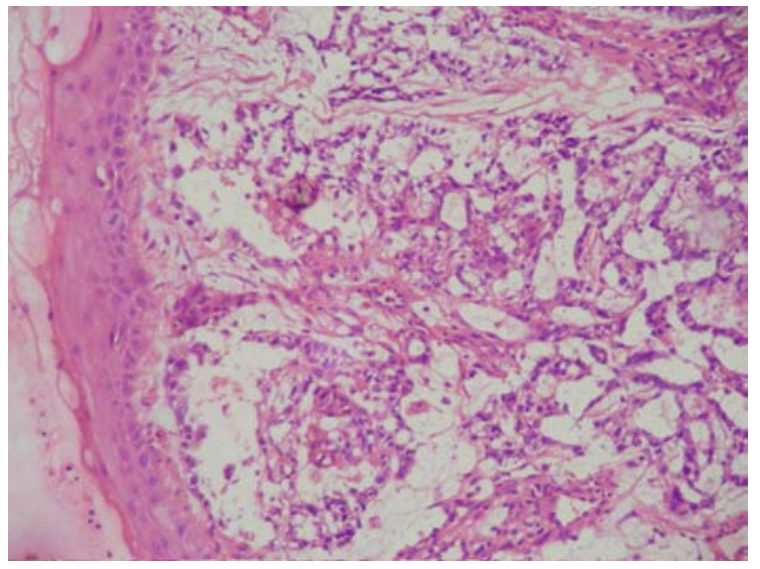

Fig 4 Yolk sac tumor present just below the vaginal stratified squamous epithelial lining (low power view - 10x). 


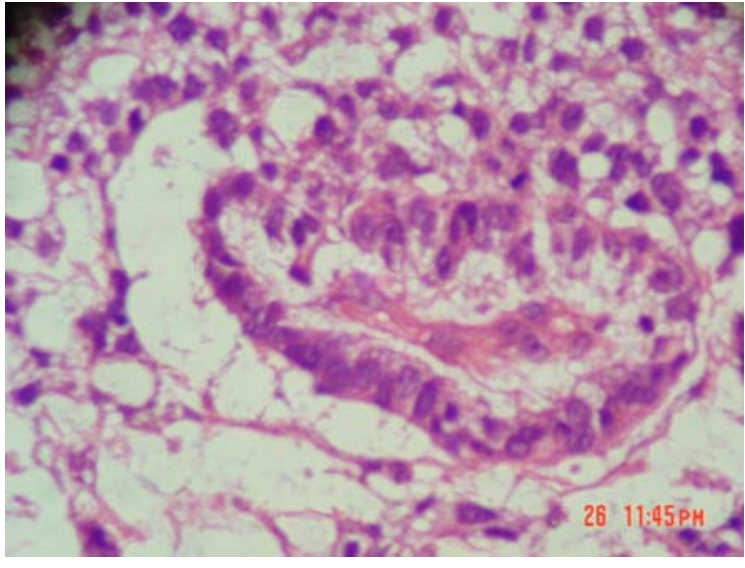

Fig 5 Glomeruloid body formed by blood vessel surrounded by tumor cells (high power view - 40x)

Immunohistochemical markers include AFP, cytokeratin, alpha-1-antitrypsin and focal positivity are also seen for CD 30. There is absence of progesterone and estrogen receptors, and $\beta-\mathrm{HCG}$ is also negative in most cases. ${ }^{5,6}$

Untreated patients may die within 2-4 months after diagnosis. ${ }^{4}$ However, with surgery and chemotherapy, long term survival has been seen in up to $60 \%$ of cases. ${ }^{1,2,6}$

\section{Conclusion}

Yolk sac tumors are rare but highly malignant tumors among the tumors of the vagina. In young girls presenting with vaginal bleeding or mass, a possibility of such a tumor should be ruled out. FNAC, histopathology, ultrasonography and AFP levels are some of the modalities of detection. Early detection and therapy is important because it is a highly aggressive tumor that shows good response to surgery and chemotherapy.

\section{References}

1. Kumar V, Kini P, Vepakomma D, M B. Vaginal endodermal sinus tumor. Indian J Pediatrics 2005;72: 797-798

2. M. Arora, R. Shrivastav, M. Jaiprakash. A rare germ-cell tumor site: vaginal endodermal sinus tumor. Pediatric Surgery International 2002; 18 : 521-523

3. Deshmukh C, Bakshi A, Bhagwat R, Kurkure P. Yolk Sac Tumor of Vagina. Indian J Pediatrics 2005 ;72: 367-367

4. Afroz N, Khan N, Chana RS. Cytodiagnosis of Yolk Sac Tumor. Indian Journal of Pediatrics 2004; 71: 939-942

5. Nogales F, Talerman A, Kubik-Huch et al. Germ Cell Tumors. In: Fattaneh A, Tavassoli A, Devilee, eds. WHO Classification of Tumors: Pathology and Genetics of the Breast and Female Genital Organs. IARC Press, Lyon, 2003: 165-166

6. Kurman R, Norris H, Wilkinson, E. Atlas of Tumor Pathology: Tumors of Cervix, Vagina, and Vulva. Third Series, Fascicle 4. AFIP, 1992: 168-169 